\title{
Ascoli-type theorems in the cone metric space setting
}

\section{Antonio Boccuto ${ }^{1}$ and Xenofon Dimitriou ${ }^{2 *}$}

"Correspondence:

xenofon11@gmail.com;

dxenof@windtools.gr

${ }^{2}$ Department of Mathematics,

University of Athens,

Panepistimiopolis, Athens, 15784

Greece

Full list of author information is

available at the end of the article

\begin{abstract}
We give some necessary and sufficient conditions for (global) continuity of the limit of a pointwise convergent net of cone metric space-valued functions, defined on a Hausdorff topological space, in terms of weak filter exhaustiveness. In this framework, we prove some Ascoli-type theorems, considering also possibly asymmetric and extended real-valued distance functions. Furthermore, we pose some open problems. MSC: Primary 26E50; 28A12; 28A33; 28B10; 28B15; 40A35; 46G10; 54A20; 54A40; secondary 06F15; 06F20; 06F30; 22A10; 28A05; 40G15; 46G12; 54H11; 54H12; 47H10
\end{abstract}

Keywords: lattice group; cone metric space; (free) filter; global continuity; filter convergence; (weak) filter exhaustiveness; Ascoli theorem; Lipschitz metric

\section{Introduction}

In the literature there have been several studies about cone metric spaces, namely abstract structures endowed with a distance function taking values in an ordered vector or a normed space, which includes in particular metric semigroups, whose an example is the set of fuzzy numbers, which is not a group (see for instance [1-5]). These structures are closely related with order vector spaces endowed with abstract convergences satisfying suitable axioms, but in which in general convergence of subsequences of convergent sequences is not required, like filter convergence (see also [6-12]). A comprehensive historical survey on main properties of these structures and several (recent) results about abstract convergences, distances with values in normed, solid or Hausdorff topological vector spaces and fixed point theorems, which have several applications to differential, functional and stochastic equations and reconstruction of signals can be found in [1329]. In this paper we investigate some properties of continuity of the limit of a net of functions, taking values in cone metric spaces, in terms of weak filter exhaustiveness, extending earlier results proved in [30-32], and relate filter exhaustiveness with filter uniform convergence (on compact subsets). Moreover, we give some Ascoli-type theorems for lattice group-valued functions defined on metric or topological spaces, extending previous results proved in [30] (see also [33]), and consider also asymmetric distances (see also [32, 34]) and extended real-valued distances, like Lipschitz metrics, dealing with functions which are not necessarily contractions and extending earlier results proved for real-valued or metric space-valued functions in [30, 32] and [35], respectively. We present some examples to support the results obtained in our setting. Asymmetric distance has different applications in several branches of mathematics and in physics, for example in gradient

@2014 Boccuto and Dimitriou; licensee Springer. This is an Open Access article distributed under the terms of the Creative Commons Attribution License (http://creativecommons.org/licenses/by/2.0), which permits unrestricted use, distribution, and reproduction in any medium, provided the original work is properly cited. 
flow models (see also [34] and the bibliography therein), and is related also with the study of several semicontinuity properties of functions (see for instance [36]). Observe that extended Lipschitz metrics are complete and extended Lipschitz metric convergence is, in general, strictly stronger than uniform convergence on bounded sets. Moreover, extended Lipschitz metrics are equivalent with the supremum metrics when the topological space $X$ in which the involved functions are defined is bounded and uniformly discrete (in its own metric), and they are also equivalent with the Sherbert and Weaver metrics when $X$ is just bounded (see also [35, 37-39]). Furthermore, note that, since in lattice groups the order convergence is in general not generated by any topology (see also [40]), in our context it is not advisable to deal with concepts like closedness and compactness in terms of topologies. So we formulate the corresponding notions directly in the setting of convergence and in terms of function nets, including the classical concepts as particular cases and giving some relations between filter pointwise convergence and filter uniform convergence on compact sets. In the literature, there have been several recent studies about abstract Ascoli-type theorems, which extend earlier results given in [41]. In [42], different Ascoli-type theorems are proved, in connection with various kinds of convergence and exhaustiveness of function nets. In [30] and [43] these convergences, together with the concept of exhaustiveness, are considered in the filter/ideal context, and in this setting some Ascoli-type theorems for real-valued functions are extended. Some other versions of the Ascoli theorem can be found, for instance, in [34, 44-46]. Our approach is direct, simple and easy to handle in the context of our considered structures, that is, when it is dealt with nets of functions taking values in cone metric spaces, defined in general Hausdorff topological spaces and with filter exhaustiveness instead of metric spaces and equicontinuity, respectively, and it allows us to give direct necessary and sufficient conditions. We consider symmetric or asymmetric distances with values in lattice groups and use the tool of (weak) filter exhaustiveness in connection with (global) continuity of the limit function and uniform convergence on compact sets. One of the main used methods is to use some kinds of convergence of suitable subnets of the given net to deduce some compactness properties. This is given in a very abstract context, comparing two kinds of compactness for function nets, and after a particular case is presented, using compactness of suitable sets, properties of convergence and boundedness in metric spaces and the Tychonoff theorem. Furthermore we consider Lipschitz-type metrics using completeness properties an a 'total boundedness' argument in terms of subsequence, without using a topological approach. Finally, we pose some open problems.

\section{Preliminaries}

We begin with some fundamental properties of convergence and continuity in the lattice group context.

A nonempty set $\Lambda=(\Lambda, \geq)$ is said to be directed iff $\geq$ is a reflexive and transitive binary relation on $\Lambda$, such that for any two elements $\lambda_{1}, \lambda_{2} \in \Lambda$ there is $\lambda_{0} \in \Lambda$ with $\lambda_{0} \geq \lambda_{1}$ and $\lambda_{0} \geq \lambda_{2}$.

A cone metric space is a nonempty set $R$ endowed with a function $\rho: R \times R \rightarrow Y$, where $Y$ is a Dedekind complete lattice group, satisfying the following axioms:

- $\rho\left(r_{1}, r_{2}\right) \geq 0$ and $\rho\left(r_{1}, r_{2}\right)=0$ if and only if $r_{1}=r_{2}$;

- $\rho\left(r_{1}, r_{2}\right)=\rho\left(r_{2}, r_{1}\right)$ (symmetric property);

- $\rho\left(r_{1}, r_{3}\right) \leq \rho\left(r_{1}, r_{2}\right)+\rho\left(r_{2}, r_{3}\right)$ (triangular property), for all $r_{j} \in R, j=1,2,3$ 
(see also [11,12]). Such a function $\rho$ will be called a distance function. If $\rho$ satisfies only the first and the third of the above axioms, but not necessarily the symmetric property, then we say that $\rho$ is an asymmetric distance function and that $(R, \rho)$ is an asymmetric cone metric space (for literature on the asymmetric case, see also [34] and the related bibliography). Note that any Dedekind complete $(\ell)$-group $Y$ is a cone metric space: indeed, it is enough to take $\rho\left(y_{1}, y_{2}\right)=\left|y_{1}-y_{2}\right|, y_{1}, y_{2} \in Y$ (the absolute value).

When $R$ is a semigroup and $Y=\mathbb{R}$, we say that $R$ is a metric semigroup. An example of metric semigroup which is not a group is the set $L(\mathbb{R})$ of the fuzzy numbers (see also $[1$, $4])$.

Example 2.1 Let $T$ any arbitrary nonempty set, $R=\{f: T \rightarrow \mathbb{R}, f$ is bounded $\}$, and fix a positive real number $a \neq 1$. For every $t \in T$ and $f_{1}, f_{2} \in R$, put

$$
d_{t}\left(f_{1}(t), f_{2}(t)\right)= \begin{cases}f_{2}(t)-f_{1}(t), & \text { if } f_{1}(t) \leq f_{2}(t), \\ a\left(f_{2}(t)-f_{1}(t)\right), & \text { if } f_{1}(t)>f_{2}(t)\end{cases}
$$

and set $d\left(f_{1}, f_{2}\right)=\sup _{t \in T} d_{t}\left(f_{1}(t), f_{2}(t)\right)$. It is not difficult to check that $d$ is an asymmetric distance function (see also [34]).

Let $R$ be a (possibly asymmetric) cone metric space and $Y$ be its associated Dedekind complete $(\ell)$-group. A sequence $\left(\sigma_{p}\right)_{p}$ in $Y$ is called an $(O)$-sequence iff it is decreasing and $\bigwedge_{p} \sigma_{p}=0$. A net $\left(x_{\lambda}\right)_{\lambda \in \Lambda}$ in $R$ (that is an indexed system of elements of $R$ such that the index set $\Lambda$ is directed) is forward order convergent or forward $(O)$-convergent (resp. backward order convergent or backward $(O)$-convergent) to $x \in R$ iff there exists an $(O)$ sequence $\left(\sigma_{p}\right)_{p}$ in $Y$ such that for every $p \in \mathbb{N}$ there is $\lambda \in \Lambda$ with $\rho\left(x, x_{\zeta}\right) \leq \sigma_{p}$ (resp. $\left.\rho\left(x_{\zeta}, x\right) \leq \sigma_{p}\right)$ for all $\zeta \in \Lambda, \zeta \geq \lambda$. We say that $\left(x_{\lambda}\right)_{\lambda \in \Lambda}$ order converges or $(O)$-converges to $x \in R$ iff it is both forward and backward $(O)$-convergent to $x$, and in this case we will write (O) $\lim _{\lambda \in \Lambda} x_{\lambda}=x$.

Let $X$ be a Hausdorff topological space. A function $f: X \rightarrow R$ is said to be forward (resp. backward) continuous at a point $x \in X$ iff there exists an $(O)$-sequence $\left(\sigma_{p}\right)_{p}$ in $Y$ (depending on $x$ ) such that for every $p \in \mathbb{N}$ there is a neighborhood $U_{x}$ of $x$ with $\rho(f(x), f(z)) \leq \sigma_{p}$ (resp. $\rho(f(z), f(x)) \leq \sigma_{p}$ ) whenever $z \in U_{x}$.

A function $f: X \rightarrow R$ is globally forward (resp. backward) continuous on $X$ iff there is an $(O)$-sequence $\left(\sigma_{p}\right)_{p}$ in $Y$ such that for any $p \in \mathbb{N}$ and $x \in X$ there is a neighborhood $U_{x}$ of $x$ with $\rho(f(x), f(z)) \leq \sigma_{p}$ (resp. $\rho(f(z), f(x)) \leq \sigma_{p}$ ) for each $z \in U_{x}$. We say that $f \in$ $R^{X}$ is (globally) continuous on $X$ iff it is both (globally) forward and (globally) backward continuous on $X$.

We now recall some basic notions on ideals and filters.

Let $\Lambda$ be any nonempty set, and $\mathcal{P}(\Lambda)$ be the class of all subsets of $\Lambda$. A family of sets $\mathcal{I} \subset \mathcal{P}(\Lambda)$ is called an ideal of $\Lambda$ iff $A \cup B \in \mathcal{I}$ whenever $A, B \in \mathcal{I}$ and for each $A \in \mathcal{I}$ and $B \subset A$ we get $B \in \mathcal{I}$. A class of sets $\mathcal{F} \subset \mathcal{P}(\Lambda)$ is a filter of $\Lambda$ iff $A \cap B \in \mathcal{F}$ for all $A, B \in \mathcal{F}$ and for every $A \in \mathcal{F}$ and $B \supset A$ we have $B \in \mathcal{F}$.

An ideal $\mathcal{I}$ (resp. a filter $\mathcal{F}$ ) of $\Lambda$ is said to be non-trivial iff $\mathcal{I} \neq \emptyset$ and $\Lambda \notin \mathcal{I}$ (resp. $\mathcal{F} \neq \emptyset$ and $\emptyset \notin \mathcal{F})$.

Let $(\Lambda, \geq)$ be a directed set. A non-trivial ideal $\mathcal{I}$ of $\Lambda$ is said to be $(\Lambda)$-admissible iff $\Lambda \backslash M_{\lambda} \in \mathcal{I}$ for each $\lambda \in \Lambda$, where $M_{\lambda}:=\{\zeta \in \Lambda: \zeta \geq \lambda\}$. 
A non-trivial filter $\mathcal{F}$ of $\Lambda$ is $(\Lambda)$-free iff $M_{\lambda} \in \mathcal{F}$ for every $\lambda \in \Lambda$.

Given an ideal $\mathcal{I}$ of $\Lambda$, we call dual filter of $\mathcal{I}$ the family $\mathcal{F}=\{\Lambda \backslash I: I \in \mathcal{I}\}$. In this case we say that $\mathcal{I}$ is the dual ideal of $\mathcal{F}$ and we get $\mathcal{I}=\{\Lambda \backslash F: F \in \mathcal{F}\}$.

When $\Lambda=\mathbb{N}$ endowed with the usual order, the $(\mathbb{N})$-admissible ideals and the (N)-free filters are called simply admissible ideals and free filters, respectively. The filter $\mathcal{F}_{\text {cofin }}$ is the filter of all subsets of $\mathbb{N}$ whose complement is finite, and its dual ideal $\mathcal{I}_{\text {fin }}$ is the family of all finite subsets of $\mathbb{N}$. The filter $\mathcal{F}_{\text {st }}$ is the filter of all subsets of $\mathbb{N}$ having asymptotic density 1 , while its dual ideal $\mathcal{I}_{\text {st }}$ is the family of all subsets of $\mathbb{N}$, having null asymptotic density. Note that $\mathcal{F}_{\text {st }}$ is a $P$-filter, namely a filter $\mathcal{F}$ of $\mathbb{N}$ such that for every sequence $\left(A_{n}\right)_{n}$ in $\mathcal{F}$ there is another sequence $\left(B_{n}\right)_{n}$ in $\mathcal{F}$, such that the symmetric difference $A_{n} \Delta B_{n}$ is finite for all $n \in \mathbb{N}$ and $\bigcap_{n=1}^{\infty} B_{n} \in \mathcal{F}$ (see also [33, 47]).

A nonempty family $\mathcal{B}^{\prime} \subset \mathcal{P}(\Lambda)$ is said to be a filter base of $\Lambda$ iff for every $A, B \in \mathcal{B}^{\prime}$ there is an element $C \in \mathcal{B}^{\prime}$ with $C \subset A \cap B$. Note that, if $\mathcal{B}^{\prime}$ is a filter base of $\Lambda$, then the family $\mathcal{F}=\left\{A \subset \Lambda\right.$ : there is $B \in \mathcal{B}^{\prime}$ with $\left.B \subset A\right\}$ is a filter of $\Lambda$. We call it the filter generated by $\mathcal{B}^{\prime}$.

If $\mathcal{B}^{\prime}=\left\{M_{\lambda}: \lambda \in \Lambda\right\}$, then $\mathcal{B}^{\prime}$ is a filter base of $\Lambda$, and the filter $\mathcal{F}_{\Lambda}$ generated by $\mathcal{B}^{\prime}$ is a $(\Lambda)$-free filter of $\Lambda$ (see also [48]).

Let $\mathcal{F}$ be a $(\Lambda)$-free filter of $\Lambda$ and choose $\bar{y} \in R$. A net $\left(s_{\lambda}\right)_{\lambda \in \Lambda}$ in $R$ is said to be $\mathcal{F}$ forward bounded (resp. $\mathcal{F}$-backward bounded) with respect to $\bar{y}$ iff there is $k_{0} \in Y, k_{0} \geq 0$ such that $\left\{\lambda \in \Lambda: \rho\left(\bar{y}, s_{\lambda}\right) \leq k_{0}\right\} \in \mathcal{F}$ (resp. $\left\{\lambda \in \Lambda: \rho\left(s_{\lambda}, \bar{y}\right) \leq k_{0}\right\} \in \mathcal{F}$ ). We say that $\left(s_{\lambda}\right)_{\lambda}$ is $\mathcal{F}$-bounded with respect to $\bar{y}$ iff it is both $\mathcal{F}$-forward and $\mathcal{F}$-backward bounded with respect to $\bar{y}$, and that $\left(s_{\lambda}\right)_{\lambda}$ is bounded (resp. forward bounded, backward bounded) iff it is $\mathcal{F}_{\text {cofin }}$-bounded (resp. $\mathcal{F}_{\text {cofin }}$-forward bounded, $\mathcal{F}_{\text {cofin }}$-backward bounded).

We now give the fundamental notions of filter convergence and related topics in the cone metric space setting. Without loss of generality, we consider the symmetric case. Analogously it is possible to deal with the corresponding 'backward' and 'forward' concepts in the asymmetric case (see also [34]).

A net $\left(x_{\lambda}\right)_{\lambda \in \Lambda}$ in a cone metric space $R(O \mathcal{F})$-converges to $x \in R\left(\right.$ shortly, $(O \mathcal{F}) \lim _{\lambda} x_{\lambda}=x$ ) iff there exists an $(O)$-sequence $\left(\sigma_{p}\right)_{p}$ in $Y$ with $\left\{\lambda \in \Lambda: \rho\left(x_{\lambda}, x\right) \leq \sigma_{p}\right\} \in \mathcal{F}$ for each $p \in \mathbb{N}$. A net $\left(x_{\lambda}\right)_{\lambda \in \Lambda}$ in $R$ is $(O \mathcal{F})$-Cauchy iff there is an $(O)$-sequence $\left(\tau_{p}\right)_{p}$ in $Y$ such that for every $p \in \mathbb{N}$ there is $F_{0} \in \mathcal{F}$ with $\rho\left(x_{\lambda}, x_{\xi}\right) \leq \tau_{p}$ for each $\lambda, \xi \in F_{0}$. Note that, since $R$ is Dedekind complete, a net $\left(f_{\lambda}\right)_{\lambda}$ in $R$ is $(O \mathcal{F})$-convergent if and only if it is $(O \mathcal{F})$-Cauchy (see also [33, 48]).

Let $\Xi$ be any nonempty set. We say that a family $\left\{\left(x_{\lambda, \xi}\right)_{\lambda}: \xi \in \Xi\right\}$ in $R(O \mathcal{F})$-converges to $x_{\xi} \in R$ uniformly with respect to $\xi \in \Xi$ (shortly, $(U O \mathcal{F})$-converges to $x_{\xi}$ ) as $\lambda$ varies in $\Lambda$ iff there is an $(O)$-sequence $\left(v_{p}\right)_{p}$ in $Y$ with

$$
\left\{\lambda \in \Lambda: \bigvee_{\xi \in \Xi} \rho\left(x_{\lambda, \xi}, x_{\xi}\right) \leq v_{p}\right\} \in \mathcal{F} \quad \text { for every } p \in \mathbb{N}
$$

A family $\left\{\left(x_{\lambda, \xi}\right)_{\lambda}: \xi \in \Xi\right\}(R O \mathcal{F})$-converges to $x_{\xi} \in R$ (as $\lambda$ varies in $\Lambda$ ) iff there exists an $(O)$-sequence $\left(\sigma_{p}\right)_{p}$ in $Y$ such that for each $p \in \mathbb{N}$ and $\xi \in \Xi$ we get $\left\{\lambda \in \Lambda: \rho\left(x_{\lambda, \xi}, x_{\xi}\right) \leq\right.$ $\left.\sigma_{p}\right\} \in \mathcal{F}$. By $(R O)$-convergence we will denote the $\left(R O \mathcal{F}_{\Lambda}\right)$-convergence. Observe that, when $R=Y=\mathbb{R},(R O \mathcal{F})$-convergence coincides with usual filter convergence (see also [8, 9, 33]). 
Let $x \in X$. A net $f_{\lambda}: X \rightarrow R, \lambda \in \Lambda$, is said to be $\mathcal{F}$-exhaustive at $x$ iff there is an $(O)$ sequence $\left(\sigma_{p}\right)_{p}$ such that for any $p \in \mathbb{N}$ there exist a neighborhood $U$ of $x$ and a set $F \in \mathcal{F}$ such that for each $\lambda \in F$ and $z \in U$ we have $\rho\left(f_{\lambda}(z), f_{\lambda}(x)\right) \leq \sigma_{p}$.

A net $f_{\lambda}: X \rightarrow R, \lambda \in \Lambda$, is weakly $\mathcal{F}$-exhaustive at $x$ iff there is an $(O)$-sequence $\left(\sigma_{p}\right)_{p}$ such that for each $p \in \mathbb{N}$ there is a neighborhood $U$ of $x$ such that for every $z \in U$ there is $F_{z} \in \mathcal{F}$ with $\rho\left(f_{\lambda}(z), f_{\lambda}(x)\right) \leq \sigma_{p}$ whenever $\lambda \in F_{z}$.

We say that $f_{\lambda}: X \rightarrow R, \lambda \in \Lambda$, is (weakly) $\mathcal{F}$-exhaustive on $X$ iff it is (weakly) $\mathcal{F}$ exhaustive at every $x \in X$ with respect to a single (O)-sequence, independent of $x \in X$.

Similarly as above it is possible to formulate the concepts of (weak) $\mathcal{F}$-forward (backward) exhaustiveness (see also [32]).

Of course the concepts of (weak, forward, backward) filter exhaustiveness can be given also analogously for sequences of functions, by taking $\Lambda=\mathbb{N}$ with the usual order.

In the next section we will see that, in general, the notion of weak $\mathcal{F}$-exhaustiveness is strictly weaker than that of $\mathcal{F}$-exhaustiveness (for the case $R=Y=\mathbb{R}$ see also [30, Remark 2.8]).

\section{The main results}

We now give, in the context of filter convergence and lattice groups, a necessary and sufficient condition under which the limit of a pointwise convergent net $\left(f_{\lambda}\right)_{\lambda}$ is (globally) continuous, extending [31, Proposition 17] to nets of lattice group-valued functions.

Theorem 3.1 Under the same above notations and assumptions, let $\mathcal{F}$ be a $(\Lambda)$-free filter of $\Lambda$, fix $x \in X$, and suppose that $f_{\lambda}: X \rightarrow R, \lambda \in \Lambda,(R O \mathcal{F})$-converges to $f: X \rightarrow R$ on $X$ with respect to a single $(O)$-sequence $\left(\sigma_{p}^{*}\right)_{p}$ in $Y$. Then the following are equivalent:

(i) $\left(f_{\lambda}\right)_{\lambda}$ is weakly $\mathcal{F}$-exhaustive at $x$;

(ii) $f$ is continuous at $x$.

Proof (i) $\Rightarrow$ (ii) Let $\left(\sigma_{p}\right)_{p}$ be an $(O)$-sequence in $Y$ associated with weak $\mathcal{F}$-exhaustiveness of $\left(f_{\lambda}\right)_{\lambda}$ at $x$, and pick $p \in \mathbb{N}$. By hypothesis, there exists a neighborhood $U_{x}$ of $x$, related with weak $\mathcal{F}$-exhaustiveness. Fix arbitrarily $z \in U_{x}$. There is a set $F_{1} \in \mathcal{F}$ (depending on $x$ and $z$ ) with $\rho\left(f_{\lambda}(x), f_{\lambda}(z)\right) \leq \sigma_{p}$ for all $\lambda \in F_{1}$. Moreover, thanks to $(R O \mathcal{F})$-convergence with respect to the $(O)$-sequence $\left(\sigma_{p}^{*}\right)_{p}$, there exists a set $F_{2} \in \mathcal{F}$ (depending on $x$ and $z$ ) with $\rho\left(f_{\lambda}(z), f(z)\right) \leq \sigma_{p}^{*}$ and $\rho\left(f_{\lambda}(x), f(x)\right) \leq \sigma_{p}^{*}$ whenever $\lambda \in F_{2}$. Thus for every $\lambda \in F_{1} \cap F_{2}$ we get

$$
\rho(f(x), f(z)) \leq \rho\left(f(x), f_{\lambda}(x)\right)+\rho\left(f_{\lambda}(x), f_{\lambda}(z)\right)+\rho\left(f_{\lambda}(z), f(z)\right) \leq 2 \sigma_{p}^{*}+\sigma_{p} .
$$

(ii) $\Rightarrow$ (i) Since $f$ is continuous at $x$, there exists an $(O)$-sequence $\left(\tau_{p}\right)_{p}$ such that for each $p \in \mathbb{N}$ there is a neighborhood $U_{x}$ of $x$ with

$$
\rho(f(x), f(z)) \leq \tau_{p}
$$

whenever $z \in U_{x}$.

By $(R O \mathcal{F})$-convergence of $\left(f_{\lambda}\right)_{\lambda}$ to $f$ on $X$ with respect to the $(O)$-sequence $\left(\sigma_{p}^{*}\right)_{p}$, there is a set $F^{*} \in \mathcal{F}$ with

$$
\rho\left(f_{\lambda}(x), f(x)\right) \leq \sigma_{p}^{*} \quad \text { and } \quad \rho\left(f_{\lambda}(z), f(z)\right) \leq \sigma_{p}^{*}
$$


for each $\lambda \in F^{*}$. From (1) and (2) we obtain

$$
\rho\left(f_{\lambda}(x), f_{\lambda}(z)\right) \leq \rho\left(f_{\lambda}(x), f(x)\right)+\rho\left(f_{\lambda}(z), f(z)\right)+\rho(f(x), f(z)) \leq 2 \sigma_{p}^{*}+\tau_{p}
$$

for every $\lambda \in F^{*}$. From (3) we get the existence of an $(O)$-sequence $\left(v_{p}\right)_{p}$ with the property that for every $z \in U_{x}$ there is a set $F^{*} \in \mathcal{F}$ (depending on $x$ and $z$ ) with $\rho\left(f_{\lambda}(x), f_{\lambda}(z)\right) \leq v_{p}$ whenever $\lambda \in F^{*}$. Thus the net $\left(f_{\lambda}\right)_{\lambda}$ is weakly $\mathcal{F}$-exhaustive at $x$. This ends the proof.

Analogously as Theorem 3.1, it is possible to prove the following.

Theorem 3.2 Under the same notations and assumptions as in Theorem 3.1, suppose that $f_{\lambda}: X \rightarrow R, \lambda \in \Lambda,(R O \mathcal{F})$-converges to $f: X \rightarrow R$ on $X$ with respect to a single $(O)$-sequence $\left(\sigma_{p}^{*}\right)_{p}$ in $Y$. Then the following are equivalent:

(i) $\left(f_{\lambda}\right)_{\lambda}$ is weakly $\mathcal{F}$-exhaustive on $X$;

(ii) $f$ is globally continuous on $X$.

We now use Theorem 3.2 to show that, in general, $\mathcal{F}$-exhaustiveness is strictly stronger than weak $\mathcal{F}$-exhaustiveness.

Example 3.3 Let $\mathcal{F}$ be any fixed free filter of $\mathbb{N}, X=[-1,1]$ be endowed with the usual distance, $\Sigma$ be the $\sigma$-algebra of all Borel subsets of $X, v$ be the Lebesgue measure on $X$, and $R$ be the space of all bounded $v$-measurable functions on $X$, with identification up to $v$ null sets. Note that order convergence in $R$ coincides with almost everywhere convergence dominated by an element of $R$, which does not have a topological nature (see also [40]). For each real number $a$, let $\underline{a}$ the function which associates to every element $x \in X$ the constant $a$.

We consider the sequence $f_{n}: X \rightarrow R, n \in \mathbb{N}$, defined as follows:

$$
f_{n}(x)= \begin{cases}\underline{0}, & \text { if } x \in[-1,0], \\ \underline{n}, & \text { if } x=\frac{1}{n}, \\ \frac{1}{n}, & \text { if } x \in(0,1], x \neq \frac{1}{n} .\end{cases}
$$

It is easy to see that $\left(f_{n}\right)_{n}(O \mathcal{F})$-converges pointwise to $\underline{0}$ with respect to an $(O)$-sequence independent of $x \in X$ (for example, $\sigma_{p}=\frac{1}{p}, p \in \mathbb{N}$ ) and thus, by Theorem 3.2, $\left(f_{n}\right)_{n}$ is weakly $\mathcal{F}$-exhaustive on $[-1,1]$. On the other hand, it is not hard to see that, if $\left(\sigma_{p}\right)_{p}$ is any $(O)$ sequence in $R$, then there is a positive real number $M_{0}$ with $\sigma_{p}(x) \leq M_{0}$ for every $p \in \mathbb{N}$ and $x \in X$. In correspondence with $M_{0}$, for each set $F \in \mathcal{F}$ there is an integer $n_{0} \in F, n_{0}>M_{0}$. So we get $f_{n_{0}}\left(\frac{1}{n_{0}}\right)=\underline{n}_{0}$, and hence $f_{n_{0}}\left(\frac{1}{n_{0}}\right) \not \leq \sigma_{1}$. From this it follows that $\left(f_{n}\right)_{n}$ is not $\mathcal{F}$ exhaustive at 0 . Moreover, it is not difficult to check that $\left(f_{n}\right)_{n}$ is $\mathcal{F}$-backward exhaustive at 0 , but not $\mathcal{F}$-forward exhaustive at 0 .

The following two propositions extend [49, Proposition 3.5] and will be useful in the sequel.

Proposition 3.4 Let $\mathcal{F}, X, R$ be as in Theorem 3.2, $f_{\lambda}: X \rightarrow R, \lambda \in \Lambda$, be a function net, $\mathcal{F}$-exhaustive on $X$ and $(R O \mathcal{F})$-convergent to $f \in R^{X}$ on $X$.

Then $f$ is globally continuous on $X$, and the net $\left(f_{\lambda}\right)_{\lambda}(U O \mathcal{F})$-converges on every compact subset $C \subset X$ with respect to a single $(O)$-sequence, independent of $C$. 
Proof Global continuity of $f$ on $X$ follows from Theorem 3.2.

Let now $\left(\sigma_{p}\right)_{p},\left(\sigma_{p}^{*}\right)_{p},\left(\tau_{p}\right)_{p}$ be three $(O)$-sequences in $Y$, related with $\mathcal{F}$-exhaustiveness of $\left(f_{\lambda}\right)_{\lambda},(R O \mathcal{F})$-convergence on $X$ and global continuity of $f$ on $X$ respectively, let $C \subset X$ be any compact set, and choose arbitrarily $p \in \mathbb{N}$ and $x \in C$. As $\left(f_{\lambda}\right)_{\lambda}$ is $\mathcal{F}$-exhaustive at $x$ and $f$ is globally continuous, there exist $F_{x} \in \mathcal{F}$ and an open neighborhood $U_{x}$ of $x$, with

$$
\rho\left(f_{\lambda}(z), f_{\lambda}(x)\right) \leq \sigma_{p} \quad \text { and } \quad \rho(f(z), f(x)) \leq \tau_{p}
$$

for each $\lambda \in F_{x}$ and $z \in U_{x}$. By compactness of $C$ there is a finite family $\left\{U_{x_{1}}, U_{x_{2}}, \ldots, U_{x_{k}}\right\}$, with $x_{j} \in X$ for every $j \in[1, k]$, whose union contains $C$. Since $\left(f_{\lambda}\right)_{\lambda}(R O \mathcal{F})$-converges to $f$ on $X$, in correspondence with $p \in \mathbb{N}$ and $x_{1}, \ldots, x_{k}$ there is a set $F_{0} \in \mathcal{F}$ with

$$
\rho\left(f_{\lambda}\left(x_{j}\right), f\left(x_{j}\right)\right) \leq \sigma_{p}^{*} \quad \text { for each } j \in[1, k] \text { and } \lambda \in F_{0} .
$$

Let $F:=F_{0} \cap\left(\bigcap_{j=1}^{k} F_{x_{j}}\right)$ : note that $F \in \mathcal{F}$. Pick arbitrarily $z \in C$ : there exists at least $j \in[1, k]$ with $z \in U_{x_{j}}$. Then from (4) and (5) we get

$$
\rho\left(f_{\lambda}(z), f(z)\right) \leq \rho\left(f_{\lambda}(z), f_{\lambda}\left(x_{j}\right)\right)+\rho\left(f_{\lambda}\left(x_{j}\right), f\left(x_{j}\right)\right)+\rho\left(f\left(x_{j}\right), f(z)\right) \leq \sigma_{p}+\sigma_{p}^{*}+\tau_{p}
$$

for each $\lambda \in F$. This ends the proof.

Proposition 3.5 Let $\mathcal{F}, X, R$ be as above. If $f_{\lambda}: X \rightarrow R, \lambda \in \Lambda$, is a net of functions, globally continuous with respect to a single $(O)$-sequence independent of $\lambda$ and $(U O \mathcal{F})$-convergent to $f \in R^{X}$ on $X$, then $f$ is globally continuous and $\left(f_{\lambda}\right)_{\lambda}$ is $\mathcal{F}$-exhaustive on $X$.

Proof We begin with proving global continuity of $f$ on $X$. Let $x \in X,\left(\sigma_{p}\right)_{p}$ and $\left(\tau_{p}\right)_{p}$ be two $(O)$-sequences, related with $(U O \mathcal{F})$-convergence of $\left(f_{\lambda}\right)_{\lambda}$ to $f$ and global continuity of the $f_{\lambda}$ 's, respectively, and fix arbitrarily $x \in X$ and $p \in \mathbb{N}$. By hypothesis there is a set $F \in \mathcal{F}$ with $\rho\left(f_{\lambda}(z), f(z)\right) \leq \sigma_{p}$ for each $\lambda \in F$ and $z \in X$. Fix $\bar{\lambda} \in F$. By global continuity of $f_{\bar{\lambda}}$ there are a neighborhood $U$ of $x$ with $\rho\left(f_{\bar{\lambda}}(z), f_{\bar{\lambda}}(x)\right) \leq \tau_{p}$ for each $z \in U$. Thus for such $z$ 's we get

$$
\begin{aligned}
\rho(f(z), f(x)) & \leq \rho\left(f(z), f_{\bar{\lambda}}(z)\right)+\rho\left(f_{\bar{\lambda}}(z), f_{\bar{\lambda}}(x)\right)+\rho\left(f_{\bar{\lambda}}(x), f(x)\right) \\
& \leq 2 \sigma_{p}+\tau_{p},
\end{aligned}
$$

namely global continuity of $f$ with respect to the $(O)$-sequence $\left(v_{p}\right)_{p}$, where $v_{p}:=2 \sigma_{p}+\tau_{p}$, $p \in \mathbb{N}$.

We now prove $\mathcal{F}$-exhaustiveness of the net $\left(f_{\lambda}\right)_{\lambda}$ on $X$. Choose arbitrarily $x \in X$. By global continuity of $f$ with respect to $\left(v_{p}\right)_{p}$, in correspondence with $p \in \mathbb{N}$ and $x \in X$ there is a neighborhood $U_{x}$ of $x$ with $\rho(f(z), f(x)) \leq v_{p}$ whenever $z \in U_{x}$. By $(U O \mathcal{F})$-convergence of $\left(f_{\lambda}\right)_{\lambda}$ to $f$ on $X$ with respect to $\left(\sigma_{p}\right)_{p}$, there is $F^{*} \in \mathcal{F}$ with

$$
\rho\left(f_{\lambda}(z), f(z)\right) \leq \sigma_{p} \quad \text { and } \quad \rho\left(f_{\lambda}(x), f(x)\right) \leq \sigma_{p} \quad \text { for all } \lambda \in F^{*} .
$$

From (6) and (7) we get

$$
\rho\left(f_{\lambda}(z), f_{\lambda}(x)\right) \leq \rho\left(f_{\lambda}(z), f(z)\right)+\rho\left(f_{\lambda}(x), f(x)\right)+\rho(f(z), f(x)) \leq 2 \sigma_{p}+v_{p}
$$

for every $\lambda \in F^{*}$ and $z \in U_{x}$. This ends the proof. 
Remark 3.6 Proceeding analogously as above, it is possible to see that Theorems 3.1, 3.2 and Propositions 3.4, 3.5 hold even when the distance function $\rho$ does not satisfy necessarily symmetric property, and $(R O \mathcal{F})-((U O \mathcal{F})$-) convergence, (weak) $\mathcal{F}$-exhaustiveness and continuity are replaced by $(R O \mathcal{F})-((U O \mathcal{F})$-) forward (backward) convergence, (weak) $\mathcal{F}$-forward (backward) exhaustiveness and forward (backward) continuity, respectively, under the hypothesis that the forward and backward convergences are equivalent.

Example 3.7 In general, the hypothesis that forward convergence implies backward convergence is essential. Indeed, let $X=[0,1]$ be endowed with the usual distance, $R=$ $[0,1] \times[0,1]$ be endowed with the following distance function:

$$
d\left(\left(y_{1}^{\prime}, y_{2}^{\prime}\right),\left(y_{1}^{\prime \prime}, y_{2}^{\prime \prime}\right)\right)= \begin{cases}0, & \text { if }\left(y_{1}^{\prime}, y_{2}^{\prime}\right)=\left(y_{1}^{\prime \prime}, y_{2}^{\prime \prime}\right) \\ \sqrt{\left(y_{1}^{\prime}-y_{1}^{\prime \prime}\right)^{2}+\left(y_{2}^{\prime}-y_{2}^{\prime \prime}\right)^{2},} & \text { if } y_{1}^{\prime} \leq y_{1}^{\prime \prime} \text { and } y_{1}^{\prime \prime}>0 \\ 1, & \text { otherwise. }\end{cases}
$$

Observe that $(R, d)$ is an asymmetric metric space (see also [34, Example 5.10]). Let $f_{n}: X \rightarrow R, n \in \mathbb{N}$, be defined by setting $f_{n}(x)=\left(\frac{1}{n}, x\right), n \in \mathbb{N}, x \in[0,1]$. Note that $d\left((0, x),\left(\frac{1}{n}, x\right)\right)=\frac{1}{n}$ and $d\left(\left(\frac{1}{n}, x\right),(0, x)\right)=1$. Thus, it is not difficult to check that for any free filter $\mathcal{F}$ of $\mathbb{N}$ the sequence $\left(f_{n}(x)\right)_{n} \mathcal{F}$-forward converges uniformly on $[0,1]$ to the function $f:[0,1] \rightarrow R$ defined by $f(x)=(0, x), x \in[0,1]$ and that the $\mathcal{F}$-forward limit is unique, while the $\mathcal{F}$-backward limit of $\left(f_{n}(x)\right)_{n}$ does not exist in $R$ for any $x \in[0,1]$. Moreover, since $d((0, x),(0,0))=d((0,0),(0, x))=1$ for each $x \in(0,1]$, we find that $f$ is neither forward nor backward continuous at 0 . Furthermore, as

$$
d\left(f_{n}(x), f_{n}(0)\right)=d\left(\left(\frac{1}{n}, x\right),\left(\frac{1}{n}, 0\right)\right)=x=d\left(\left(\frac{1}{n}, 0\right),\left(\frac{1}{n}, x\right)\right)=d\left(f_{n}(x), f_{n}(0)\right)
$$

for every $x \in[0,1]$, it follows that for any free filter $\mathcal{F}$ of $\mathbb{N}$ the sequence $\left(f_{n}(x)\right)_{n}$ is both $\mathcal{F}$-forward and $\mathcal{F}$-backward exhaustive at 0 .

We now give some versions of Ascoli-type theorems in the context of lattice groups and filter exhaustive nets, extending earlier results proved in [30] and [32]. Note that in our context, since we deal with abstract structures which are not necessarily by a topology, it will be advisable to deal with suitable notions of 'filter closedness' and 'filter compactness' in relation with convergences, which are not necessarily generated by a Hausdorff topology. For example, note that in the space $L^{0}([0,1], \Sigma, v)$ of all measurable functions on $[0,1]$ with respect to the $\sigma$-algebra $\Sigma$ of all Borel subsets of $[0,1]$ and the Lebesgue measure $v$, with identification up to $v$-null sets, order convergence coincides with almost everywhere convergence, which does not have a topological nature. Moreover, there exist Dedekind complete vector lattices which do not have any Hausdorff compatible vector topology, for which every bounded monotone increasing sequence converges to its supremum (see for instance [40]).

Given a directed set $\Lambda$, a $(\Lambda)$-free filter $\mathcal{F}$ of $\Lambda$, a topological space $X$, a cone metric space $R$ and a nonempty set $\Phi \subset R^{X}$, we say that $\Phi$ is $(R O \mathcal{F})$-compact (resp. $(c \mathcal{F})$-compact) iff every net $\left(f_{\lambda}\right)_{\lambda \in \Lambda}$ in $\Phi$ admits a subnet $\left(f_{\lambda_{\kappa}}\right)_{\kappa \in \Lambda},(R O \mathcal{F})$-convergent to an element $f \in$ $\Phi$ (resp. (UOF) -convergent to an element $f \in \Phi$ on every compact subset $C \subset X$ with respect to a single $(O)$-sequence independent of $C)$. We say that $\Phi$ is $(R O \mathcal{F})$-closed iff $f \in \Phi$ 
whenever $\left(f_{\lambda}\right)_{\lambda}$ is a net in $\Phi,(R O \mathcal{F})$-convergent to $f \in R^{X}$. The $(R O \mathcal{F})$-closure of $\Phi$ is the set of the functions $f \in R^{X}$, having a net $\left(f_{\lambda}\right)_{\lambda}$ in $\Phi,(R O \mathcal{F})$-convergent to $f$. Analogously as above, it is possible to formulate the notions of $(c \mathcal{F})$-closedness and of $(c \mathcal{F})$-closure. Note that $\Phi$ is $(R O \mathcal{F})$-closed (resp. $(c \mathcal{F})$-closed) if and only if it coincides with its $(R O \mathcal{F})$ closure (resp. $(c \mathcal{F})$-closure).

When $R=\mathbb{R},(R O \mathcal{F})$-convergence coincides with $\mathcal{F}$-pointwise convergence, and hence we denote the related above concepts by $\mathcal{F}$-compactness, $\mathcal{F}$-closedness and $\mathcal{F}$-closure, respectively.

We now are in a position to give the following abstract Ascoli-type theorem.

Theorem 3.8 Under the same notations and hypotheses as above, if $\Phi \subset \Psi \subset R^{X}$, where $\Phi$ is $(c \mathcal{F})$-closed and $\Psi$ is $(R O \mathcal{F})$-compact, and

$\left(\mathrm{H}^{\prime}\right)$ every $(R O \mathcal{F})$-convergent net $\left(h_{\lambda}\right)_{\lambda \in \Lambda}$ in $\Phi$ has a subnet $\left(h_{\lambda_{\xi}}\right)_{\xi \in \Lambda},(R O \mathcal{F})$-convergent (in $R^{X}$ ) and $\mathcal{F}$-exhaustive on $X$,

then $\Phi$ is $(c \mathcal{F})$-compact.

Moreover, if $\Phi$ is $(c \mathcal{F})$-compact, then $\Phi$ satisfies condition $\left(\mathrm{H}^{\prime}\right)$.

Proof We begin with the first part. Let $\Phi \subset R^{X}$ be $(c \mathcal{F})$-closed, and $\left(f_{\lambda}\right)_{\lambda \in \Lambda}$ be a net in $\Phi$. Since $\Phi \subset \Psi$ and $\Psi$ is $(R O \mathcal{F})$-compact, $\left(f_{\lambda}\right)_{\lambda}$ has a $(R O \mathcal{F})$-convergent subnet $\left(f_{\lambda_{\kappa}}\right)_{\kappa \in \Lambda}$. By condition $\left(\mathrm{H}^{\prime}\right)$, this subnet has a sub-subnet $\left(f_{\lambda_{\kappa_{\zeta}}}\right)_{\zeta \in \Lambda},(R O \mathcal{F})$-convergent to a function $f \in$ $R^{X}$ and $\mathcal{F}$-exhaustive on $X$. By Proposition 3.4, the net $\left(f_{\lambda_{K_{\zeta}}}\right)_{\zeta} \mathcal{F}$-converges to $f$ uniformly on every compact subset $C \subset X$, with respect to an $(O)$-sequence independent of $C$. Since $\Phi$ is $(c \mathcal{F})$-closed, then $f \in \Phi$. Therefore, $\Phi$ is $(c \mathcal{F})$-compact.

We now turn to the last part. Let $\Phi$ be $(c \mathcal{F})$-compact and $\left(f_{\lambda}\right)_{\lambda \in \Lambda}$ be a $(R O \mathcal{F})$-convergent net in $\Phi$. Then it admits a subnet $\left(f_{\lambda_{\kappa}}\right)_{\kappa \in \Lambda},(U O \mathcal{F})$-convergent to a function $f \in R^{X}$ on every compact subset $C \subset X$ with respect to an $(O)$-sequence, independent of $C$. By Proposition 3.5, we find that $\left(f_{\lambda_{\kappa}}\right)_{\kappa \in \Lambda}(R O \mathcal{F})$-converges to $f$ and is $\mathcal{F}$-exhaustive on every compact set $C$ with respect to a single $(O)$-sequence, and hence, by arbitrariness of $C$, it $(R O \mathcal{F})$ converges to $f$ and is $\mathcal{F}$-exhaustive on $X$. This ends the proof.

Remark 3.9 Observe that, in general, condition $\left(\mathrm{H}^{\prime}\right)$ is strictly weaker than equicontinuity (see also [42, Remark 3.2.17]).

A consequence of Theorem 3.8 is the following (see also [32]).

Theorem 3.10 Let $\left(X, d_{X}\right)$ and $\left(R, d_{R}\right)$ be asymmetric metric spaces, such that $d_{X}$ and $d_{R}$ are real-valued distance functions, $\bar{y}$ be a fixed element of $R, \mathcal{F}$ be any free filter of $\mathbb{N}$ and suppose that

(3.10.1) each subset of $R, \mathcal{F}$-closed and $\mathcal{F}$-forward bounded with respect to $\bar{y}$, is $\mathcal{F}$-compact.

Let $\Phi \subset R^{X}$ be such that

(3.10.2) every sequence $\left(f_{n}\right)_{n}$ in $\Phi$, pointwise convergent in $R^{X}$, has a $\mathcal{F}_{\text {cofin-exhaustive }}$ subsequence;

(3.10.3) every sequence $\left(f_{n}\right)_{n}$ in $\Phi$ has a subsequence $\left(f_{n_{r}}\right)_{r}, \mathcal{F}$-pointwise forward bounded in $R$ with respect to $\bar{y}$. 
Then every sequence $\left(f_{n}\right)_{n}$ in $\Phi$ admits a subsequence, uniformly convergent on every compact subset $C \subset X$ in the usual sense.

Proof Choose arbitrarily $x \in X$, set $\Phi_{x}:=\{f(x): f \in \Phi\}$, and let $\bar{\Phi}_{x}$ be the closure of $\Phi_{x}$ in $R$. We claim that $\bar{\Phi}_{x}$ is compact in $R$. Indeed, let $y \in \bar{\Phi}_{x}$. There exists a sequence $\left(y_{n}\right)_{n}$ in $\Phi_{x}$, convergent to $y$ with respect to $d_{R}$. So, in correspondence with $\varepsilon=1$ there is a natural number $n_{0} \in \mathbb{N}$ with $d_{R}\left(y, y_{n}\right) \leq 1$ whenever $n \geq n_{0}$. Moreover, there is a sequence $\left(f_{n}\right)_{n}$ in $\Phi$ such that $f_{n}(x)=y_{n}$ for each $n \in \mathbb{N}$. By (3.10.3), there exist a subsequence $\left(f_{n_{r}}\right)_{r}$ of $\left(f_{n}\right)_{n}$, a positive real number $k_{x}$ and a set $F_{x} \in \mathcal{F}$ with $d_{R}\left(\bar{y}, y_{n_{r}}\right) \leq k_{x}$ whenever $r \in F_{x}$. Thus there exists $r_{0} \in \mathbb{N}$ with

$$
d_{R}(\bar{y}, y) \leq d_{R}\left(\bar{y}, y_{n_{r_{0}}}\right)+d_{R}\left(y_{n_{r_{0}}}, y\right) \leq k_{x}+1
$$

getting forward boundedness of $\bar{\Phi}_{x}$ and hence $\mathcal{F}$-compactness of $\bar{\Phi}_{x}$, thanks to the hypotheses. This implies that $\bar{\Phi}_{x}$ is also compact: indeed, by proceeding analogously as in [50, Proposition 2.4], it is possible to prove that every $\mathcal{F}$-convergent sequence in an asymmetric metric space $(X, d)$ has a subsequence, convergent in the usual sense. As $\bar{\Phi}_{x}$ is compact in $R$, then, by virtue of the Tychonoff theorem, the set $\prod_{x \in X} \bar{\Phi}_{x}$ is compact in $R^{X}$ with respect to the pointwise convergence. Since $\Phi \subset \prod_{x \in X} \bar{\Phi}_{x}$, then we find that every sequence $\left(f_{n}\right)_{n}$ in $\Phi$ has a subsequence $\left(f_{n_{k}}\right)_{k}$, pointwise convergent to a suitable function $h \in R^{X}$. From (3.10.2) and Theorem 3.8 used with $Y=\mathbb{R}, \Lambda=\mathbb{N}$ and $\mathcal{F}=\mathcal{F}_{\text {cofin, }}$ where the roles of $\Phi$ and $\Psi$ are played by the $(c \mathcal{F})$-closure $\bar{\Phi}$ of $\Phi$ and $\prod_{x \in X} \bar{\Phi}_{x}$ respectively, we get the assertion.

Note that Theorem 3.10 extends [30, Theorem 3.7] and [32, Theorem 3.4]. Indeed, we get the following.

Corollary 3.11 Under the same hypotheses and notations as in Theorem 3.10, let $\mathcal{F}$ be a P-filter of $\mathbb{N}$. Let $\Phi \subset R^{X}$ satisfy (3.10.1), (3.10.2) and be such that

(3.11.1) every sequence in $\Phi$, pointwise convergent in $R^{X}$, has a $\mathcal{F}$-exhaustive subsequence.

Then every sequence $\left(f_{n}\right)_{n}$ has a subsequence, uniformly convergent on $X$ in the usual sense.

Proof Let $\left(f_{n}\right)_{n}$ be any function sequence in $\Phi$. Arguing analogously as in the proof of Theorem 3.10, we see that $\left(f_{n}\right)_{n}$ has a subsequence $\left(f_{n_{k}}\right)_{k}$, pointwise convergent in the usual sense to a function $h \in R^{X}$. By (3.11.1), $\left(f_{n_{k}}\right)_{k}$ has a $\mathcal{F}$-forward exhaustive subsequence, say $\left(h_{n}\right)_{n}$. Since $\mathcal{F}$ is a $P$-filter, proceeding analogously as in [30, Lemma 3.6], it is possible to see that $\left(h_{n}\right)_{n}$ has a $\mathcal{F}_{\text {cofin }}$-forward exhaustive subsequence. The assertion follows from Theorem 3.10 .

Example 3.12 In general, in Theorem 3.10, the condition (3.10.1) cannot be dropped. Indeed, let $X,(R, d)$ and $f_{n}: X \rightarrow R, n \in \mathbb{N}$, be as in Example 3.7. Note that $(R, d)$ is bounded and closed (with respect to itself), and also $\mathcal{F}$-closed and $\mathcal{F}$-bounded for any free filter $\mathcal{F}$ of $\mathbb{N}$. Let $A=\left\{x_{n}:=\left(0, \frac{1}{n}\right): n \in \mathbb{N}\right\}$ : since $d\left(\left(0, \frac{1}{n}\right),(0, b)\right)=1$ whenever $b \neq \frac{1}{n}$ and $d\left(\left(0, \frac{1}{n}\right),(a, b)\right) \geq a$ for every $a \in(0,1], b \in[0,1]$ and $n \in \mathbb{N}$, it is not difficult to see that, for each free filter $\mathcal{F}$ of $\mathbb{N}$, every subsequence $\left(x_{n_{r}}\right)_{r}$ of $\left(x_{n}\right)_{n}$ does not admit $\mathcal{F}$-forward limit 
as $r \rightarrow+\infty$. From this it follows that $(R, d)$ is not $\mathcal{F}$-forward compact and hence it does not fulfil condition (3.10.1). Let now $\Phi:\left\{f_{n}: n \in \mathbb{N}\right\}$ : it is not hard to check that the conditions (3.10.2) and (3.10.3) are satisfied. Note that, as seen in Example 3.7, the sequence $\left(f_{n}\right)_{n}$ does not have any subsequence, uniformly (backward) convergent in the usual sense, and so the thesis of Theorem 3.10 is false.

We now give some versions of abstract Ascoli-type theorems with respect to Lipschitztype metrics. For a related literature, we refer to [35] and the bibliography therein.

Let $(X, d)$ be a metric space endowed with a real-valued distance function, $R$ be a Dedekind complete vector lattice, $Y=R$ endowed with the absolute value, and let us add to $R$ an extra element $+\infty$, satisfying the properties analogous to those of the element $+\infty$ of the extended real line. We say that $f: X \rightarrow R$ is Lipschitz iff there is a positive element $M \in R$ with $\left|f\left(x_{1}\right)-f\left(x_{2}\right)\right| \leq d\left(x_{1}, x_{2}\right) M$ whenever $x_{1}, x_{2} \in X$, and in this case we set

$$
\Pi(f):=\bigvee\left\{\frac{\left|f\left(x_{1}\right)-f\left(x_{2}\right)\right|}{d\left(x_{1}, x_{2}\right)}: x_{1}, x_{2} \in X: x_{1} \neq x_{2}\right\}
$$

If $f: X \rightarrow R$ is not Lipschitz, then we put $\Pi(f):=+\infty$. Note that, even if $X$ is a compact metric space, $R=\mathbb{R}$ and $f: X \rightarrow \mathbb{R}$ is continuous, it may happen that $\Pi(f)=+\infty$ : indeed, it is enough to take $f(x)=x^{1 / 2}, x \in[0,1]$.

We now fix a point $x_{0} \in X$ and consider the following extended metric:

$$
d_{L}\left(f_{1}, f_{2}\right):=\left|f_{1}\left(x_{0}\right)-f_{2}\left(x_{0}\right)\right| \vee \Pi\left(f_{1}-f_{2}\right), \quad f_{1}, f_{2}: X \rightarrow R .
$$

Given a directed set $(\Lambda, \geq)$ and a $(\Lambda)$-free filter $\mathcal{F}$ of $\Lambda$, we say that

$$
(\mathcal{F}) \lim _{\lambda \in \Lambda} d_{L}\left(f_{\lambda}, f\right)=0 \quad \text { or } \quad\left(\mathcal{F} d_{L}\right) \lim _{\lambda \in \Lambda} f_{\lambda}=f
$$

iff there is an $(O)$-sequence $\left(\sigma_{p}\right)_{p}$, with

$$
\left\{\lambda \in \Lambda: d_{L}\left(f_{\lambda}, f\right) \leq \sigma_{p}\right\} \in \mathcal{F} \quad \text { for every } p \in \mathbb{N}
$$

In this case, we say that the net $\left(f_{\lambda}\right)_{\lambda}\left(\mathcal{F} d_{L}\right)$-converges to $f$. The net $\left(f_{\lambda}\right)_{\lambda}$ is $\left(\mathcal{F} d_{L}\right)$-Cauchy iff there is an $(O)$-sequence $\left(\sigma_{p}\right)_{p}$ in $R$ with the property that for every $p \in \mathbb{N}$ there is $F \in \mathcal{F}$ with $d_{L}\left(f_{\xi}, f_{\zeta}\right) \leq \sigma_{p}$ whenever $\xi, \zeta \in F$.

It is easy to check that every $\left(\mathcal{F} d_{L}\right)$-convergent net is $\left(\mathcal{F} d_{L}\right)$-Cauchy. We will prove also the converse implication. To this aim, we first give the following extension of [35, Proposition 3.5] to the setting of filters and vector lattices.

Proposition 3.13 Let $f_{\lambda}: X \rightarrow R, \lambda \in \Lambda$, be a function net, $\left(\mathcal{F} d_{L}\right)$-convergent to $f$, and $x_{0}$ be related with $d_{L}$. Then, for every $k>0,\left(f_{\lambda}\right)_{\lambda}(U O \mathcal{F})$-converges to $f$ on the set $S\left(x_{0}, k\right):=$ $\left\{x \in X: d\left(x, x_{0}\right)<k\right\}$.

Proof By hypothesis, there is an $(O)$-sequence $\left(\sigma_{p}\right)_{p}$ such that for every $p \in \mathbb{N}$ there is a set $F \in \mathcal{F}$ with

$$
\left|f_{\lambda}\left(x_{0}\right)-f\left(x_{0}\right)\right| \leq \sigma_{p} \quad \text { and } \quad\left|\left(f_{\lambda}-f\right)(x)-\left(f_{\lambda}-f\right)\left(x_{0}\right)\right| \leq d\left(x, x_{0}\right) \sigma_{p}
$$


for every $\lambda \in F$ and $x \in X$. From (10) we get

$$
\left|f_{\lambda}(x)-f(x)\right| \leq(1+k) \sigma_{p}
$$

whenever $\lambda \in F$ and $x \in S\left(x_{0}, k\right)$. This ends the proof.

Example 3.14 Observe that, in general, $\left(\mathcal{F} d_{L}\right)$-convergence is strictly stronger than $(U O \mathcal{F})$-convergence on compact sets. Indeed, let $(X, d)$ be a compact metric space, fix a point $x \in X$, and suppose that there exists at least a sequence $\left(x_{n}\right)_{n}$ in $X$, convergent to $x$ with respect to $d$ in the usual sense. Let $R$ be any Dedekind complete vector lattice, $u$ be a strictly positive element of $R$, and put $f_{n}(t)=d\left(t, x_{n}\right) u, f(t)=d(t, x) u$ for every $n \in \mathbb{N}$ and $t \in X$. It is not difficult to check that $\left|f_{n}(t)-f(t)\right|=\left|d\left(t, x_{n}\right) u-d(t, x) u\right| \leq d\left(x, x_{n}\right) u$ for any $n \in \mathbb{N}$ and $t \in X$. From this it follows that the sequence $\left(f_{n}\right)_{n}(U O \mathcal{F})$-converges on $X$ for every free filter $\mathcal{F}$ of $\mathbb{N}$. On the other hand, we get

$$
\begin{aligned}
\Pi\left(f_{n}-f\right) & \geq \frac{\left|\left(f_{n}-f\right)\left(x_{n}\right)-\left(f_{n}-f\right)(x)\right|}{d\left(x_{n}, x\right)} \\
& =\frac{\left|d\left(x_{n}, x_{n}\right) u-d\left(x, x_{n}\right) u-d\left(x_{n}, x\right) u+d(x, x) u\right|}{d\left(x_{n}, x\right)}=2 u
\end{aligned}
$$

for every $n \in \mathbb{N}$. This implies that, for any free filter $\mathcal{F}$ of $\mathbb{N}$, the sequence $\left(\Pi\left(f_{n}-f\right)\right)_{n}$ does not converge to 0 . Otherwise there exists an $(O)$-sequence $\left(\sigma_{p}\right)_{p}$ in $R$ such that $\{n \in$ $\left.\mathbb{N}: \Pi\left(f_{n}-f\right) \leq \sigma_{p}\right\} \in \mathcal{F}$ for every $p \in \mathbb{N}$, and so for each $p \in \mathbb{N}$ there is $n_{p} \in \mathbb{N}$ with $2 u \leq$ $\Pi\left(f_{n_{p}}-f\right) \leq \sigma_{p}$. By arbitrariness of $p$ it follows that $u=0$, but this is impossible. Thus $\left(\Pi\left(f_{n}-f\right)\right)_{n}$ does not $(O \mathcal{F})$-converge to 0 , and hence $\left(f_{n}\right)_{n}$ is not $\left(\mathcal{F} d_{L}\right)$-convergent to $f$ (see also [35, Example 3.1]).

As a consequence of Proposition 3.13, we prove the following completeness result, which extends [35, Proposition 3.3].

Proposition 3.15 Under the same above notations and hypotheses, let $f_{\lambda}: X \rightarrow R, \lambda \in$ $\Lambda$, be an $\left(\mathcal{F} d_{L}\right)$-Cauchy net of functions, globally continuous with respect to a single $(O)$ sequence. Then $\left(f_{\lambda}\right)_{\lambda}\left(\mathcal{F} d_{L}\right)$-converges to a globally continuous function $f: X \rightarrow R$.

Proof Let $f_{\lambda}: X \rightarrow R, \lambda \in \Lambda$, be a $\left(\mathcal{F} d_{L}\right)$-Cauchy net, and $x_{0}$ be related with $d_{L}$. Then the net $\left(f_{\lambda}\left(x_{0}\right)\right)_{\lambda}$ is $(O \mathcal{F})$-Cauchy, and so it is $(O \mathcal{F})$-convergent, since $R$ is Dedekind complete (see also [48]). Moreover, for every $\lambda, \xi \in \Lambda$ and $x \in X$ we get

$$
\begin{aligned}
\left|f_{\lambda}(x)-f_{\xi}(x)\right| & \leq\left|f_{\lambda}\left(x_{0}\right)-f_{\xi}\left(x_{0}\right)\right|+d\left(x, x_{0}\right) \Pi\left(f_{\lambda}-f_{\xi}\right) \\
& \leq\left|f_{\lambda}\left(x_{0}\right)-f_{\xi}\left(x_{0}\right)\right|+d\left(x, x_{0}\right) d_{L}\left(f_{\lambda}, f_{\xi}\right) .
\end{aligned}
$$

From (11), the hypotheses and since the net $\left(f_{\lambda}\left(x_{0}\right)\right)_{\lambda}$ is $(O \mathcal{F})$-Cauchy, it follows that for every $x \in X$ the net $\left(f_{\lambda}(x)\right)_{\lambda}$ is $(O \mathcal{F})$-Cauchy, and so $(O \mathcal{F})$-convergent. For each $x \in X$, set $f(x):=(O \mathcal{F}) \lim _{\lambda} f_{\lambda}(x)$.

We now prove that $(\mathcal{F}) \lim _{\lambda} \Pi\left(f_{\lambda}-f\right)=0$. Choose arbitrarily $p \in \mathbb{N}$. Since by hypothesis $\left(f_{\lambda}\right)_{\lambda}$ is $d_{L}$-Cauchy, there is $F \in \mathcal{F}$ with $d_{L}\left(f_{\lambda}, f_{\xi}\right) \leq \sigma_{p}$, and hence

$$
\Pi\left(f_{\lambda}-f_{\xi}\right) \leq \sigma_{p}, \quad \text { whenever } \lambda, \xi \in F
$$


From (12), since $f_{\lambda}(x)-f(x)=(O \mathcal{F}) \lim _{\xi}\left(f_{\lambda}(x)-f_{\xi}(x)\right)$ for every $x \in X$ and $\lambda \in \Lambda$, it follows that

$$
\Pi\left(f_{\lambda}-f\right) \leq \sigma_{p} \quad \text { whenever } \lambda \in F \text {. }
$$

From Propositions 3.5, 3.13 and (13) we get global continuity of $f$. This ends the proof.

The next step is to give an Ascoli-type theorem involving $d_{L}$. We say that a net $f_{\lambda}: X \rightarrow R$, $\lambda \in \Lambda$, is $\mathcal{F}$-finitely $d_{L}$-bounded iff there exists a finite number $q$ of globally continuous functions $h_{1}, \ldots, h_{q} \in R^{X}$, of elements $r_{1}, \ldots, r_{q} \in R$, of sets $E_{1}, \ldots, E_{q}$ with $\Lambda=\bigcup_{j=1}^{q} E_{j}$, and a set $F \in \mathcal{F}$ such that $d_{L}\left(f_{\lambda}, h_{j}\right) \leq r_{j}$ for every $j \in[1, q]$ and whenever $\lambda \in F \cap E_{j}$.

Theorem 3.16 Let $X$ be a metric space. If $\Phi \subset \Psi \subset R^{X}$, where $\Phi$ is $(c \mathcal{F})$-closed and $\Psi$ is $(R O F)$-compact, and if we assume that

(3.16.1) every $(R O \mathcal{F})$-convergent net in $\Phi$ is $\mathcal{F}$-finitely $d_{L}$-bounded,

then $\Phi$ is $(c \mathcal{F})$-compact.

Proof Choose arbitrarily a net $\left(f_{\lambda}\right)_{\lambda \in \Lambda}$. By $(R O \mathcal{F})$-compactness of $\Psi,\left(f_{\lambda}\right)_{\lambda \in \Lambda}$ has a subnet $\left(f_{\lambda_{\zeta}}\right)_{\zeta \in \Lambda},(R O \mathcal{F})$-convergent to a function $f \in R^{X}$. By $\left(\mathrm{H}^{\prime}\right),\left(f_{\lambda_{\zeta}}\right)_{\zeta \in \Lambda}$ is $\mathcal{F}$-finitely $d_{L^{-}}$ bounded. Pick arbitrarily $x \in X$ and let $q, F$ and $h_{j}, r_{j}, E_{j}, j \in[1, q]$, be according to $\mathcal{F}$-finite $d_{L}$-boundedness of $\left(f_{\lambda_{\zeta}}\right)_{\zeta}$. Since the $h_{j}$ 's are globally continuous, we find an $(O)$-sequence $\left(\tau_{p}\right)_{p}$ such that for each $p \in \mathbb{N}$ there is a neighborhood $U_{x}$ of $x$ with

$$
\left|h_{j}(z)-h_{j}(x)\right| \leq \tau_{p} \quad \text { whenever } z \in U_{x} \text { and } j \in[1, q] .
$$

If $\zeta \in F \cap E_{j}$ and $z \in U_{x}$, then from (14) we get

$$
\begin{aligned}
& \left|f_{\lambda_{\zeta}}(z)-f_{\lambda_{\zeta}}(x)\right| \\
& \quad=\left|\left(f_{\lambda_{\zeta}}(z)-h_{j}(z)\right)+\left(h_{j}(z)-h_{j}(x)\right)+\left(h_{j}(x)-f_{\lambda_{\zeta}}(x)\right)\right| \\
& \quad \leq\left|h_{j}(z)-h_{j}(x)\right|+\left|\left(f_{\lambda_{\zeta}}-h_{j}\right)(z)-\left(f_{\lambda_{\zeta}}-h_{j}\right)(x)\right| \\
& \quad \leq \tau_{p}+d(z, x) \Pi\left(f_{\lambda_{\zeta}}-h_{j}\right) \leq \tau_{p}+d(z, x) d_{L}\left(f_{\lambda_{\zeta}}, h_{j}\right) \leq \tau_{p}+d(z, x) r_{j},
\end{aligned}
$$

getting $\mathcal{F}$-exhaustiveness of $\left(f_{\lambda_{\zeta}}\right)_{\zeta \in \Lambda}$. By Proposition 3.4 , the net $\left(f_{\lambda_{\zeta}}\right)_{\zeta \in \Lambda}(U O \mathcal{F})$ converges to $f$ on every compact subset $C \subset X$ with respect to a single $(O)$-sequence, independent of $C$. This ends the proof.

When $R=\mathbb{R}$ and $\Lambda=\mathbb{N}$, it is possible to prove the following extension of [35, Theorem 5.1].

Theorem 3.17 Let $\mathcal{F}$ be any free filter of $\mathbb{N}, X$ be a separable metric space, $\Phi \subset \mathbb{R}^{X}$ be $\left(c \mathcal{F}_{\text {cofin }}\right)$-closed, and such that every sequence $f_{n}: X \rightarrow \mathbb{R}, n \in \mathbb{N}$, in $\Phi$ is $\mathcal{F}$-finitely $d_{L^{-}}$ bounded. Then $\Phi$ is $\left(c \mathcal{F}_{\text {cofin }}\right)$-compact.

Proof Let $\left(f_{n}\right)_{n}$ be an $\mathcal{F}$-finitely $d_{L}$-bounded sequence, $x_{0}$ be related to $d_{L}$, and $F, q, h_{j}, r_{j}$, $E_{j}, j \in[1, q]$, be associated with $\mathcal{F}$-finite $d_{L}$-boundedness of $\left(f_{n}\right)_{n}$. By arguing analogously 
as in the proof of Theorem 3.16, formula (15), it is possible to show that the sequence $f_{n}$, $n \in \mathbb{N}$, satisfies condition (3.10.1) with respect to the filter $\mathcal{F}_{\text {cofin }}$. To prove (3.10.2), observe that

$$
\begin{aligned}
& \left|f_{n}(x)-h_{j}(x)\right| \\
& \quad \leq\left|f_{n}\left(x_{0}\right)-h_{j}\left(x_{0}\right)\right|+\left|f_{n}(x)-h_{j}(x)-\left(f_{n}\left(x_{0}\right)-h_{j}\left(x_{0}\right)\right)\right| \\
& \quad \leq r_{j}+d\left(x, x_{0}\right) r_{j} \quad \text { for every } x \in X, j \in[1, q], n \in F \cap E_{j} .
\end{aligned}
$$

From (16) it follows that

$$
\left|f_{n}(x)\right| \leq \bigvee_{j \in[1, q]}\left|h_{j}(x)\right|+r_{j}+d\left(x, x_{0}\right) \bigvee_{j \in[1, q]} r_{j}
$$

for each $n \in F$ and $x \in X$. Thus the sequence $f_{n}, n \in F$, satisfies (3.10.2) with respect to $\mathcal{F}_{\text {cofin. }}$. By Theorem 3.10, the sequence $f_{n}, n \in F$, and so even the sequence $f_{n}, n \in \mathbb{N}$, has a subsequence, uniformly convergent to $f$ on every compact subset $C \subset X$ in the usual sense. By $\left(c \mathcal{F}_{\text {cofin }}\right)$-closedness of $\Phi$, it follows that $f \in \Phi$. This ends the proof.

Example 3.18 Observe that, in Theorem 3.17, in general the hypothesis of $\mathcal{F}$-finite $d_{L^{-}}$ boundedness cannot be dropped. Indeed, let $f_{n}:[-1,1] \rightarrow \mathbb{R}, n \in \mathbb{N}$, be a function sequence, such that $f_{n}\left(\left[-1,-\frac{1}{n}\right]\right)=f_{n}\left(\left[\frac{1}{n}, 1\right]\right)=0, f_{n}(0)=n$ and $f_{n}$ is linear in $\left[-\frac{1}{n}, 0\right]$ and in $\left[0, \frac{1}{n}\right]$ for each $n \in \mathbb{N}$. It is not difficult to check that for every free filter $\mathcal{F}$ of $\mathbb{N}$, for any $F \in \mathcal{F}$, for each $q \in \mathbb{N}$ and for any choice of real numbers $r_{1}, \ldots, r_{q}$, of functions $h_{1}, \ldots, h_{q}$, continuous on $[-1,1]$, and of sets $E_{1}, \ldots, E_{q}$ with $\bigcup_{j=1}^{q} E_{j}=\mathbb{N}$, there exists at least an index $\bar{j} \in[1, q]$ such that $F \cap E_{\bar{j}}$ is infinite: otherwise $F$ should be finite, but this is impossible, since $\mathcal{F}$ is a free filter of $\mathbb{N}$. Thus there is a sufficiently large integer $\bar{n} \in F \cap E_{\bar{j}}$, with

$$
\Pi\left(f_{\bar{n}}-h_{\bar{j}}\right)=\sup \left\{\frac{\left|\left(f_{\bar{n}}-h_{\bar{j}}\right)\left(x_{1}\right)-\left(f_{\bar{n}}-h_{j}\right)\left(x_{2}\right)\right|}{\left|x_{1}-x_{2}\right|}: x_{1}, x_{2} \in[-1,1]: x_{1} \neq x_{2}\right\}>r_{j}
$$

Thus, for any free filter $\mathcal{F}$ of $\mathbb{N}$, the sequence $\left(f_{n}\right)_{n}$ is not $\mathcal{F}$-finitely $d_{L}$-bounded. Furthermore, it is not hard to see that the sequence $\left(f_{n}\right)_{n}$ has no subsequence convergent uniformly on $[-1,1]$, and so the set $\Phi:=\left\{f_{n}: n \in \mathbb{N}\right\}$ is $\left(c \mathcal{F}_{\text {cofin }}\right)$-closed, but not $\left(c \mathcal{F}_{\text {cofin }}\right)$-compact (see also [35, Example 5.3]).

Remark 3.19 The results here obtained hold, even if $Y$ is a weakly $\sigma$-distributive lattice group endowed with $(D)$-convergence (see for instance [51]).

\section{Open problems}

(a) Prove similar results on (weak) filter exhaustiveness in other abstract structures.

(b) Find further versions of Ascoli-type theorems, by considering different kinds of convergences in various kinds of abstract spaces.

\section{Conclusion}

We have first given, for cone metric space function nets, a characterization of continuity of the limit function in terms of filter weak exhaustiveness and some relation between 
filter exhaustiveness and uniform convergence (on compact sets). These relations have been used in proving our main abstract Ascoli-type theorem. Indeed, the condition on existence of a (filter pointwise convergent with respect to a single order sequence and) filter exhaustive subnet is necessary and sufficient for compactness with respect to the uniform convergence of compact sets, given the compactness with respect to the pointwise convergence. In the classical case, it is possible to treat these subjects by dealing with the compact-open topology (which corresponds to convergence uniformly on compacta; see also [41]). But in a general Dedekind complete lattice group it may happen the nonexistence of Hausdorff topologies 'compatible' with the order (see also [40]), and we need an approach different from the classical one, by considering concepts like closedness and compactness by means of nets (or sequences) of functions rather than by topologies. In Theorem 3.10 and Corollary 3.11, we have extended earlier results proved in [32] and [30], including both the symmetric and the asymmetric case.

Furthermore, we have dealt with Lipschitz-type metrics and considered also extended distance-type functions, which are related with not necessarily Lipschitz functions. The main Ascoli-type results given in [35] have been extended in the lattice group setting and in the context of filter convergence and exhaustiveness (and also a concept similar to 'total boundedness' has been presented in terms of filters), giving some compactness result again with respect to uniform convergence on compact sets. We have given also some examples to support the results proved in our setting.

Competing interests

The authors declare that they have no competing interests.

Authors' contributions

All authors contributed equally and significantly in writing this article. All authors read and approved the final manuscript.

\section{Author details}

${ }^{1}$ Dipartimento di Matematica e Informatica, Universita degli Studi di Perugia, via Vanvitelli, 1, Perugia, 06123, Italy.

${ }^{2}$ Department of Mathematics, University of Athens, Panepistimiopolis, Athens, 15784, Greece.

\section{Acknowledgements}

Our thanks to the referees for their remarks, which improved the exposition of the paper.

Received: 6 June 2014 Accepted: 10 October 2014 Published: 21 Oct 2014

\section{References}

1. Boccuto, A, Candeloro, D, Sambucini, AR: Stieltjes-type integrals for metric semigroup-valued functions defined on unbounded intervals. Panam. Math. J. 17, 39-58 (2007)

2. Buckley, Jj: Fuzzy Probabilities. New Approach and Applications. Physica-Verlag, Heidelberg (2003)

3. Buckley, JJ: Fuzzy Probability and Fuzzy Sets for Web Planning. Springer, Berlin (2004)

4. Riečan, B, Neubrunn, T: Integral, Measure and Ordering. Kluwer Academic, Dordrecht (1997)

5. Vrábel, P, Vrábelová, M: $\mathcal{I}$-Convergence and $\mathcal{I}$-continuity of the fuzzy number-valued functions. Acta Math. (Nitra) 16, 237-243 (2013)

6. Bardaro, C, Boccuto, A, Dimitriou, X, Mantellini, I: Abstract Korovkin-type theorems in modular spaces and applications. Cent. Eur. J. Math. 11, 1774-1784 (2013)

7. Boccuto, A, Candeloro, D: Integral and ideals in Riesz spaces. Inf. Sci. 179, 2891-2902 (2009)

8. Boccuto, A, Dimitriou, X, Papanastassiou, N: Basic matrix theorems for $\mathcal{I}$-convergence in $(\ell)$-groups. Math. Slovaca 62 , 885-908 (2012)

9. Boccuto, A, Dimitriou, X, Papanastassiou, N: Schur lemma and limit theorems in lattice groups with respect to filters Math. Slovaca 62, 1145-1166 (2012)

10. Candeloro, D, Sambucini, AR: Filter convergence and decompositions for vector lattice-valued measures. Mediterr. J. Math. (2014). doi:10.1007/500009-014-0431-0

11. Proinov, PD: A unified theory of cone metric spaces and its applications to the fixed point theory. Fixed Point Theory Appl. 2013, Article ID 103 (2013). doi:10.1186/1687-1812-2013-103

12. Zabrejko, PP: K-Metric and K-normed linear spaces: a survey. Collect. Math. 48, 825-859 (1997)

13. Boccuto, A, Candeloro, D: Several types of equations in Riesz spaces and applications. Acta Math. (Nitra) 8, 7-21 (2005)

14. Boccuto, A, Candeloro, D: The contraction principle in Riesz spaces and applications to differential and stochastic equations. Atti Semin. Mat. Fis. Univ. Modena Reggio Emilia 55, 13-31 (2007) 
15. Kadelburg, Z, Radenović, S: A note on various types of cones and fixed point results in cone metric spaces. Asian J. Math. Appl. 2013, Article ID ama0104 (2013)

16. Cvetković, AS, Radenović, S, Simić, S, Stanić, M: Generalized $\varphi$-contraction for four mappings on cone metric spaces. Indian J. Math. Special Volume on Professor Billy Rhoades 56, 209-228 (2014)

17. Shauhan, S, Bhatnagar, S, Radenović, S: Common fixed point theorems for weakly compatible mappings in fuzzy metric spaces. Matematiche 68, 87-98 (2013). doi:10.4418/2013.68.1.8

18. Shauhan, S, Shatanawi, W, Kumar, S, Radenović, S: Existence and uniqueness of fixed points in modified intuitionistic fuzzy metric spaces. J. Nonlinear Sci. Appl. 6, 1-12 (2013)

19. Chauhan, S, Radenović, S, Imdad, M, Vetro, C: Some integral type fixed point theorems in non-Archimedean Menger PM-spaces with common property (E.A) and application of functional equations in dynamic programming. Rev. R. Acad. Cienc. Exactas Fís. Nat., Ser. A Mat. (2014). doi:10.1007/s13398-013-0142-6

20. Tran, VA, Nguyen, VD, Kadelburg, Z, Radenović, S: Various generalizations of metric spaces and fixed point theorems. Rev. R. Acad. Cienc. Exactas Fís. Nat., Ser. A Mat. (2014). doi:10.1007/s13398-014-0173-7

21. Saadati, R, Kumam, P, Jang, SY: On the tripled fixed point and tripled coincidence point theorems in fuzzy normed spaces. Fixed Point Theory Appl. 2014, Article ID 136 (2014)

22. Kadelburg, Z, Radenović, S: Coupled fixed point results under tvs-cone metric spaces and $w$-cone-distance. Adv. Fixed Point Theory 2, 29-46 (2012)

23. Radenović, S, Rhoades, BE: Fixed point theorem for two non-self mappings in cone metric spaces. Comput. Math. Appl. 57, 1701-1707 (2009)

24. Radenović, S: Common fixed points under contractive conditions in cone metric spaces. Comput. Math. Appl. 58 1273-1278 (2009)

25. Janković, S, Golubović, Z, Radenović, S: Compatible and weakly compatible mappings in cone metric spaces. Math Comput. Model. 52, 1728-1738 (2010)

26. Janković, S, Kadelburg, Z, Radenović, S: On cone metric spaces: a survey. Nonlinear Anal. 74, 2591-2601 (2011)

27. Kadelburg, Z, Radenović, S: Generalized quasicontractions in orbitally complete abstract metric spaces. Fixed Point Theory 13, 527-536 (2012)

28. Kadelburg, Z, Radenović, S, Rakočević, V: A note on equivalence of some metric and cone metric fixed point results. Appl. Math. Lett. 24, 370-374 (2011)

29. Kadelburg, Z, Radenović, S, Rakočević, V: Topological vector spaces-valued cone metric spaces and fixed point theorems. Fixed Point Theory Appl. 2010, Article ID 170253 (2010). doi:10.1155/2009/170253

30. Athanassiadou, E, Boccuto, A, Dimitriou, X, Papanastassiou, N: Ascoli-type theorems and ideal $(\alpha)$-convergence. Filomat 26, 397-405 (2012). doi:10.2298/FIL1202397A

31. Athanassiadou, E, Dimitriou, X, Papachristodoulos, C, Papanastassiou, N: Strong $(\alpha)$-convergence and ideal strong exhaustiveness of sequences of functions. Int. J. Pure Appl. Math. 80, 207-216 (2012)

32. Boccuto, A, Dimitriou, X: Asymmetric Ascoli-type theorems and filter exhaustiveness (2014). arXiv:1405.0322

33. Boccuto, A, Dimitriou, X: Convergence Theorems for Lattice Group-Valued Measures. Bentham Science Publishers, Sharjah (2014)

34. Collins, J, Zimmer, J: An asymmetric Arzelà-Ascoli theorem. Topol. Appl. 154, 2312-2322 (2007)

35. Beer, G, Hoffman, MJ: The Lipschitz metric for real-valued continuous functions. J. Math. Anal. Appl. 406, 229-236 (2013)

36. Beer, G: Semicontinuous limits of nets of continuous functions. Math. Program., Ser. B 139, 71-79 (2013)

37. Sherbert, D: Banach algebras of Lipschitz functions. Pac. J. Math. 13, 1387-1399 (1963)

38. Sherbert, D: The structure of ideals and point derivations in Banach algebras of Lipschitz functions. Transl. Am. Math. Soc. 111, 240-272 (1964)

39. Weaver, N: Lipschitz Algebras. World Scientific, Singapore (1999)

40. Luxemburg, WAJ, Zaanen, AC: Riesz Spaces. I. North-Holland, Amsterdam (1971)

41. Kelley, JL: General Topology. Van Nostrand, Princeton (1955)

42. Gregoriades, V, Papanastassiou, N: The notion of exhaustiveness and Ascoli-type theorems. Topol. Appl. 155, 1111-1128 (2008)

43. Boccuto, A, Das, P, Dimitriou, X, Papanastassiou, N: Ideal exhaustiveness, weak convergence and weak compactness in Banach spaces. Real Anal. Exch. 37, 389-410 (2012)

44. Kulkarni, SH: Arzelà-Ascoli theorem is stable. Int. J. Math. Educ. Sci. Technol. 31, 919-922 (2000)

45. Li, R, Zhong, S, Swartz, C: An improvement of the Arzelà-Ascoli theorem. Topol. Appl. 159, 2058-2061 (2012)

46. Mynard, F: A convergence-theoretic viewpoint on the Arzelà-Ascoli theorem. Real Anal. Exch. 38, 431-444 (2013)

47. Kostyrko, P, Šalát, T, Wilczyński, W: $\mathcal{I}$-Convergence. Real Anal. Exch. 26, 669-685 (2001)

48. Boccuto, A, Dimitriou, X, Papanastassiou, N: Ideal convergence and divergence of nets in $(\ell)$-groups. Czechoslov. Math. J. 62(137), 1073-1083 (2012)

49. Caserta, A, Di Maio, G, Holá, L: (Strong) weak exhaustiveness and (strong uniform) continuity. Filomat 24, 63-75 (2010)

50. Boccuto, A, Das, P, Dimitriou, X: A Schur-type theorem for $\mathcal{I}$-convergence and maximal ideals. Int. J. Pure Appl. Math. 81, 517-529 (2012)

51. Boccuto, A, Dimitriou, X, Papanastassiou, N, Wilczyński, W: Ideal exhaustiveness, continuity and $\alpha$-convergence for lattice group-valued functions. Int. J. Pure Appl. Math. 70, 211-227 (2011)

$10.1186 / 1029-242 X-2014-420$

Cite this article as: Boccuto and Dimitriou: Ascoli-type theorems in the cone metric space setting. Journal of Inequalities and Applications 2014, 2014:420 